\title{
BMJ Open Prevalence of metabolic syndrome, discrete or comorbid diabetes and hypertension in sub-Saharan Africa among people living with HIV versus HIV-negative populations: a systematic review and meta-analysis protocol
}

To cite: Todowede 00 , Sartorius B. Prevalence of metabolic syndrome, discrete or comorbid diabetes and hypertension in sub-Saharan Africa among people living with HIV versus HIV-negative populations: a systematic review and meta-analysis protocol. BMJ Open 2017;7:e016602. doi:10.1136/ bmjopen-2017-016602

- Prepublication history and additional material are available. To view these files please visit the journal online (http://dx.doi. org/10.1136/bmjopen-2017016602).

Received 27 February 2017 Revised 12 April 2017 Accepted 21 April 2017

\section{(a) CrossMark}

${ }^{1}$ Public Health Medicine, University of KwaZulu-Natal College of Health Sciences, Durban, KwaZulu-Natal, South Africa

${ }^{2}$ School of Nursing and Public Health, UKZN, Durban, KwaZuluNatal, South Africa

Correspondence to Olamide 0 Todowede; lamide. ayodele@gmail.com

\section{ABSTRACT}

Introduction Metabolic disorder and high blood pressure are common complications globally, and specifically among people living with HIV (PLHIV). Diabetes, metabolic syndrome and hypertension are major risk factors for cardiovascular diseases and their related complications. However, the burden of metabolic syndrome, discrete or comorbid diabetes and hypertension in PLHIV compared with HIV-negative population has not been quantified. This review and meta-analysis aims to compare and analyse the prevalence of these trio conditions between HIV-negative and HIV-positive populations in sub-Saharan Africa (SSA).

Methods and analysis The Preferred Reporting Items for Systematic Reviews and Meta-Analysis statement guides the methods for this study. Eligibility criteria will be published original articles (English and French language) from SSA that present the prevalence of metabolic syndrome, discrete and/or comorbid diabetes, and hypertension comparisons between PLHIV and HIVnegative populations. The following databases will be searched from January 1990 to February 2017: PubMed/ Medline, EBSCOhost, Web of Science, Google Scholar, Scopus, African Index Medicus and Cochrane Database of Systematic Reviews. Eligibility screening and data extraction will be conducted independently by two reviewers, and disagreements resolved by an independent reviewer. Methodological quality and risk of bias will be assessed for individual included studies, while meta-analysis will be used to estimate study outcomes prevalence according to subgroups. Sensitivity analysis will also be performed to further test the robustness of the findings.

Ethics and dissemination This proposed study does not require ethical approval. The results will be published as a scientific article in a peer-reviewed journal, and presented at conferences and to relevant health agencies.

Trial registration number PROSPERO registration number (CRD42016045727).

\section{INTRODUCTION/RATIONALE}

The epidemiological transition model developed by Omran argued that infectious and
Strengths and limitations of this study

- Understanding the differences in the burden of metabolic syndrome (and its subcomponents), diabetes and hypertension between HIV-positive and HIV-negative populations.

- This review contributes to informing public health actions needed for non-communicable disease (NCD) comorbidities and population health.

- Stringent adherence to the Preferred Reporting Items for Systematic Reviews and Meta-Analysis Statement guidelines.

- Inclusion of non-English-language (French) published studies and literature to increase the representativeness of the findings in the region.

- A limitation is the lack of single definition criteria of metabolic syndrome over the study period; thus, hypertension and diabetes are inclusive of the subcomponents.

parasitic diseases will decrease, while chronic and ageing-related conditions and diseases will increase, these changes being driven by social factors and lifestyles. ${ }^{12}$ The generalisation of Omran's model to low-income and middle-income countries (LMICs) is not applicable due to the increased incidence of chronic diseases, an ageing population with related health conditions and a resurgence of infectious diseases among this population. ${ }^{23}$ Frenk $e t a \hat{l}$ envisioned the protracted epidemiological transition model as being able to describe the health inequality, morbidity and mortality by social class, this being applicable to LMICs that are faced with prolonged periods of both infectious and chronic diseases. ${ }^{4}$ Sub-Saharan Africa (SSA) is also undergoing a demographic transition, with increased population size and growth, 
changing age structures, inequality, urbanisation and rural exodus. ${ }^{5}$ In addition, these countries now have the highest prevalence of HIV/AIDS, with many people being on antiretroviral treatment. ${ }^{6}$ Within this context, the prevalence of non-communicable diseases (trio) is increasing and is projected to exceed that of communicable diseases by $2030,{ }^{7}$ due to epidemiological and demographic transitions. ${ }^{8}$

The leading non-communicable conditions are cardiovascular diseases (CVD) and diabetes, with hypertension being a major risk factor. ${ }^{8}$ Diabetes is a metabolic condition that affects mainly adults around the world, specifically type 2 , which is the most prevalent, accounting for approximately $95 \%$ of all cases. ${ }^{910}$ Diabetes and hypertension are also major causes of increased morbidity, mortality and other health complications globally. ${ }^{11}{ }^{12}$ The global prevalence of hypertension is $20 \%-50 \%,{ }^{13}$ while estimates suggest that diabetes will affect approximately 642 million people by 2040 , mostly among adults of age 20 years and above. ${ }^{13-16}$ Patients with diabetes have an increased incidence of hypertension and other health risks, ${ }^{10} 17$ the predisposing risk factors being obesity, high carbohydrate and sugar diets, physical inactivity and other related factors clustering into metabolic dysfunctions. ${ }^{18} 19$

Metabolic syndrome (Mets) is the clustering of risk factors for the development of type 2 diabetes and CVD, ${ }^{20}$ which has an increasing prevalence in SSA. ${ }^{21} 22$ This condition and its risk factor represents clinical concept used to indicate pre-diabetes and prehypertension. ${ }^{23}$ Mets develops from clustering conditions and the risk factors of diabetes, hypertension and CVDs. The relationship between Mets, diabetes and hypertension is complex, as high glucose intolerance and blood pressure are criteria for Mets diagnosis, while diabetes and hypertension are discretely health conditions. Nevertheless, there are a variety of definitions for Mets, each with criteria that influence its diagnosis and complexity. ${ }^{94}$ The influence of fat redistribution, such as visceral obesity, increased waist:hip ratio and adipose tissue, is an established presentation of cardiometabolic traits. ${ }^{25}$ Obesity is a major contributor to increased glucose intolerance, high blood pressure and lipid disorders that result in metabolic dysfunction. ${ }^{26}$ However, not all overweight and obese individuals are diabetic, and not all patients with diabetes are overweight or obese. ${ }^{25}$ Abdominal obesity is a consistent marker of Mets diagnosis, ${ }^{27}$ and the prevalence of Mets among patients with diabetes and hypertension rises with an increasingly ageing population. ${ }^{28}$

These trio conditions (diabetes, hypertension and Mets) are highly prevalent among the HIV-infected population as a result of their long-term dependence on antiretroviral therapy (ART) regimen, ${ }^{29}{ }^{30}$ which has been shown to be a contributing factor to developing metabolic complications, such as lipodystrophy, dyslipidemia and insulin resistance. ${ }^{31}{ }^{32}$ Modifiable lifestyle risk factors similar to the general population are also a major contributing factor to increasing metabolic disorders. ${ }^{33}$ Hypertension is a common AIDS-unrelated condition among HIV-positive persons, with an estimated prevalence of between $4.7 \%$ and $54.4 \%$ in high-income countries and $8.7 \%-45.9 \%$ in LMICs. ${ }^{34}$ These trio conditions have emerged as one of the contributors to non-AIDS-related causes of morbidity and mortality globally. ${ }^{35}$ The causative and predisposing factors of developing these conditions are similar among all populations, regardless of HIV status, and include sociodemographic change, an aged population, globalisation, overweight, obesity and sedentary lifestyles. ${ }^{16} 3637$ Globally, non-communicable disease (NCD) comorbidities in people living with HIV (PLHIV) is high, and while its prevalence is similar to the general population that is not infected, those who are infected also have to contend with the dual burden of NCD and other infectious diseases. ${ }^{38}$

Studies have shown that metabolic conditions are more common among PLHIV due to the HIV infection itself and the ARV regimen; however, these have been done mainly in developed countries. ${ }^{39-41}$ Empirical evidence about the differential cardiometabolic traits between people infected and uninfected with HIV is limited and conflicting, especially for SSA, and a consolidated estimate will assist in assessing the need for monitoring and managing metabolic dysfunction in HIV-infected populations. ${ }^{42} \mathrm{~A}$ narrative systematic review indicated the difference in the prevalence of hypertension among HIV-positive populations in developed countries to be between $4.7 \%$ and $54.4 \%$, and ranging between $8.7 \%$ and $45.9 \%$ in LMICs. ${ }^{34}$ However, most of the studies included in the review were from developed countries, the focus being on PLHIV, with no comparative HIV-negative control groups. A review without a meta-analysis on the prevalence of Mets among PLHIV reported a 30\% mean prevalence in Africa. ${ }^{43}$ With only a few studies being from South America, Africa and Asia, the result could be an overestimation or underestimation.

Most reviews with or without meta-analysis that have explored the prevalence of diabetes, hypertension and/ or Mets have focused on PLHIV, without a comparable HIV-negative baseline. ${ }^{42} 44-47$ Moreover, data comparing the burden of NCD among the HIV-positive and HIV-negative populations (in the same setting) are limited, especially in the era of increasing longevity due to ART roll-out and the epidemiological transition taking place in SSA. The growing burden of chronic diseases (including chronic HIV) will further strain the region's weak healthcare infrastructure, resources and services, and increase healthcare expenditure in coming years. ${ }^{48}$ Understanding the burden of Mets (and its individual components) among PLHIV is essential to maintaining the gains made against acute HIV morbidity and mortality. ${ }^{38}$ This proposed review therefore attempts to unpack this multimorbidity aspect by HIV status, thereby increasing the accuracy of burden estimation, which is needed for effective healthcare system planning in HIV endemic settings. ${ }^{49}{ }^{50}$ This study will conduct a systematic review and meta-analysis specific to SSA, which bears a high dual burden due to a protracted epidemiological 
transition. The risk of metabolic disorders in HIV-positive people, compared with HIV-negative populations, is an important research priority area, ${ }^{51}$ as relevant data are limited, especially in the era of increasing access to ART in SSA. This review will attempt to highlight the unique contribution of understanding the burden of Mets (and its subcomponents) and discrete or comorbid diabetes and hypertension by comparing the prevalence between HIV-positive and HIV-negative populations in SSA, which has not been explored, to the best of our knowledge.

The aim of this study is to conduct a systematic review and meta-analysis of studies documented from 1990 to 2017 to determine the differential prevalence of Mets, discrete and/or comorbid diabetes and hypertension between HIV-positive and HIV-negative populations in SSA.

\section{Research questions}

The proposed review will seek to address the following research questions:

1. What is the prevalence of diabetes among adults in SSA with and without HIV infection?

2. What is the prevalence of hypertension among adults in SSA with and without HIV infection?

3. What is the prevalence of Mets among adults in SSA with and without HIV infection?

4. What is the prevalence of comorbid diabetes and hypertension among adults in SSA with and without HIV infection?

\section{METHODS}

\section{Eligibility criteria (inclusion and exclusion)}

\section{Inclusion criteria}

The review will include studies on the prevalence of Mets (and its subcomponents), type 2 diabetes and hypertension discretely or as comorbid conditions, and exclude those on other related cardiovascular and non-communicable diseases conditions. The following factors will apply:

1. Study designs: This review and meta-analysis will include randomised control trials, cross-sectional, case-control and cohort studies that assess the prevalence of Mets (and subcomponents), discrete and/or comorbid diabetes and hypertension among PLHIV and/or HIV-negative populations in SSA. Included studies will be those conducted with both or any of the study outcomes.

2. Study participants: Adult (age 18 years and older) human participants residing in SSA, regardless of their ethnic background.

3. Study outcome definition: Outcomes will be defined through self-report, use of antihypertensive and cholesterol-lowering and antidiabetic drugs. The WHO/IDF (International Diabetes Federation) recommendation on the definition and diagnosis of diabetes mellitus for diabetes will be used. ${ }^{52}$ The seventh report of the Joint National Committee on Prevention, Detection, Evaluation and Treatment of
High Blood Pressure will be used for hypertension diagnosis definition. ${ }^{53}$ The diagnosis criteria for Mets will be that of the IDF, ${ }^{54}$ National Cholesterol Education Program-Adult Treatment Panel III definition (NCEP ATP III), ${ }^{55}$ European Group for Study of Insulin Resistance ${ }^{56}$ and WHO criteria. ${ }^{55}$ Refer to table 1 for outcome definitions.

4. Time-period: Published and grey literature and unpublished data reported between 1 January 1990 and 28 February 2017 will be included, taking into account changes in the definition of diabetes and Mets over this period.

5. Study settings: Community or population-based settings, health facilities settings within rural and urban areas of SSA will be included.

6. Study languages: All studies reported in English and French languages within any SSA country will be considered. The inclusion of French-language articles will increase the precision of combined estimates of effect size, study result generalisation and applicability ${ }^{57}$ and enhance the study coverage and robustness across SSA. A French-speaking person will assess studies published in French to ensure that the content and results are not over-rated or under-rated, after which they will be translated into English.

7. HIV status: Studies considering the outcome for PLHIVs who are on antiretroviral treatment and/or are treatment-naïve will be included, as well as those relating to HIV-negative populations.

\section{Exclusion criteria}

The following factors will apply:

1. Study design: case series/studies, reviews, commentaries and other publications without primary data.

2. Study participants: studies conducted among African populations residing in other continents.

3. Study outcome definition: studies with no Mets criteria or definition, and with different stages of hypertension progression will be excluded.

4. Study languages: studies published in languages other than English and French.

5. Study outcome results: studies lacking prevalence rates and data to compute it after consultation with the author. Duplicate publications from the same studies will be excluded, while those with outcome results published in more than one journal will be reviewed as one study, and relevant information will be collated comprehensively and authors will be contacted to validate data extracted.

\section{Source of information and search strategies}

This review will be guided and written by the Preferred Reporting Items for Systematic Reviews and Meta-Analysis Protocols (PRISMA-P) 2015 statement, as indicated in table $2{ }^{58}$ Eligible published articles on the prevalence of diabetes, hypertension and/or/with Mets among 


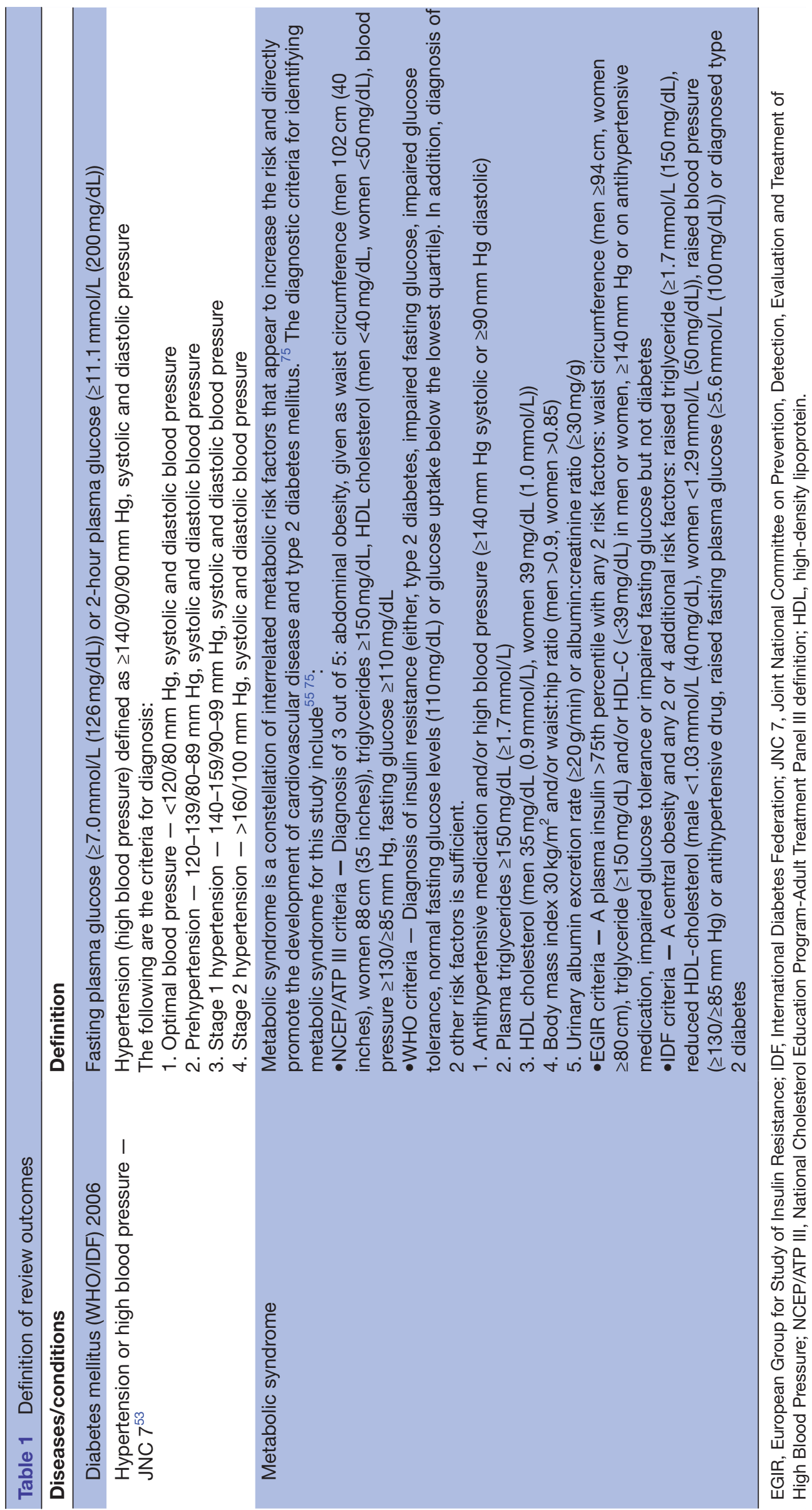




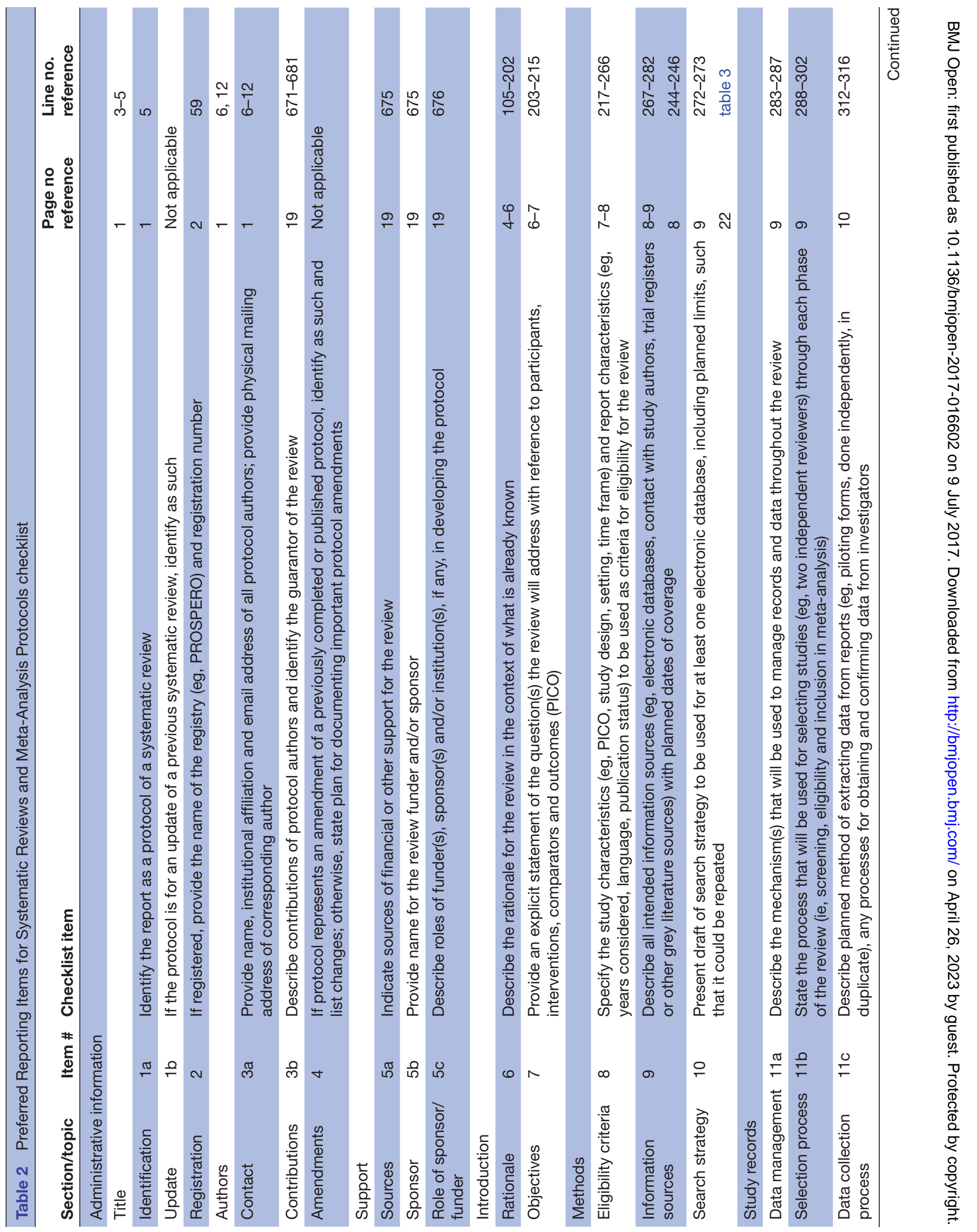




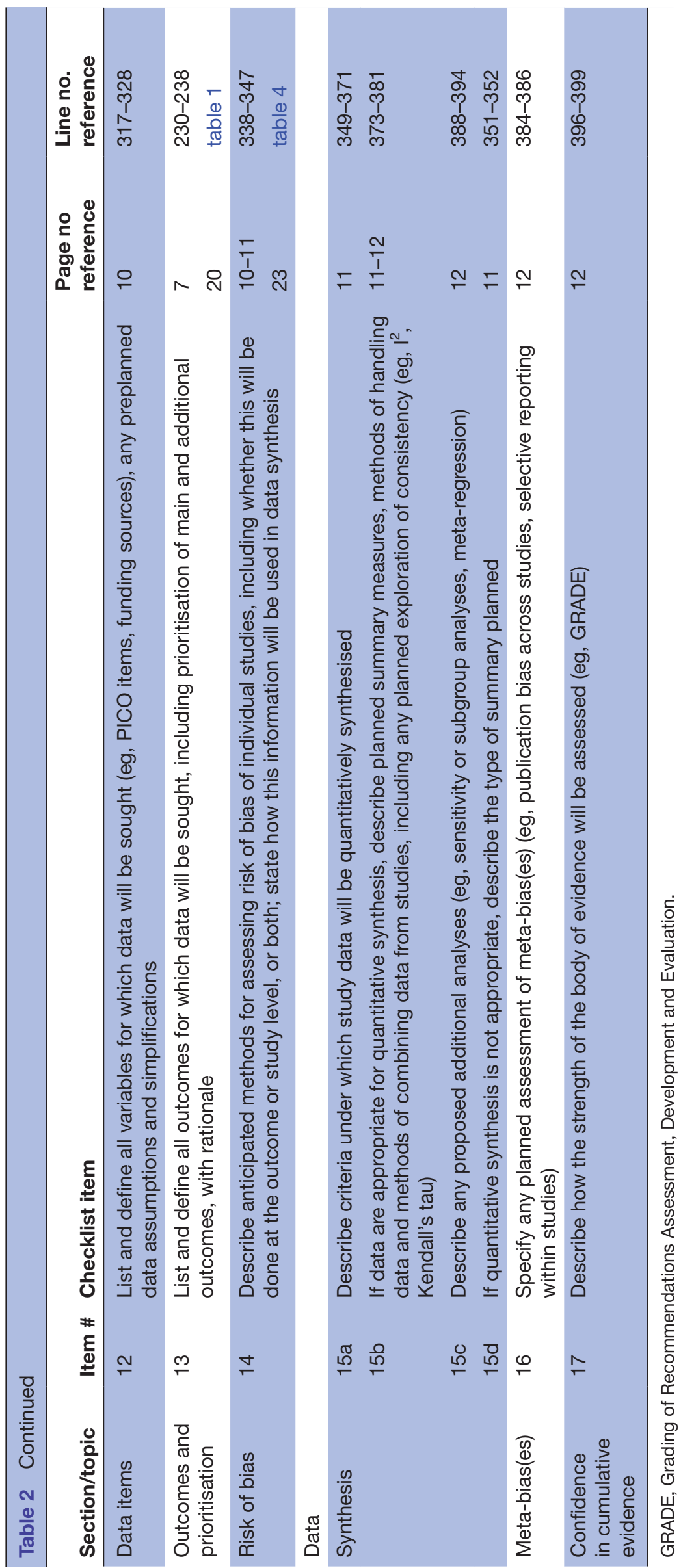

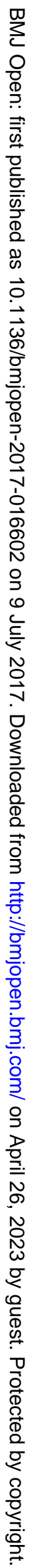




\begin{tabular}{|c|c|c|}
\hline Search & Search terms & $\begin{array}{l}\text { Number } \\
\text { of hits }\end{array}$ \\
\hline \#1 & Metabolic syndrome OR syndrome X OR insulin resistance syndrome & \\
\hline \#3 & $\begin{array}{l}\text { Type } 2 \text { diabetes mellitus OR type } 2 \text { diabetes OR diabetes Mellitus OR non-insulin dependent diabetes } \\
\text { OR adult onset diabetes }\end{array}$ & \\
\hline \#4 & $\begin{array}{l}\text { Human Immunodeficiency Virus OR Acquired Immune Deficiency Syndrome Virus OR AIDS Virus OR } \\
\text { HIV Seronegativities OR Seronegativity, HIV OR HIV Seropositivities OR Seropositivity, HIV }\end{array}$ & \\
\hline \#5 & \#1 OR \#2 OR \#3 AND \#4 & \\
\hline \#6 & 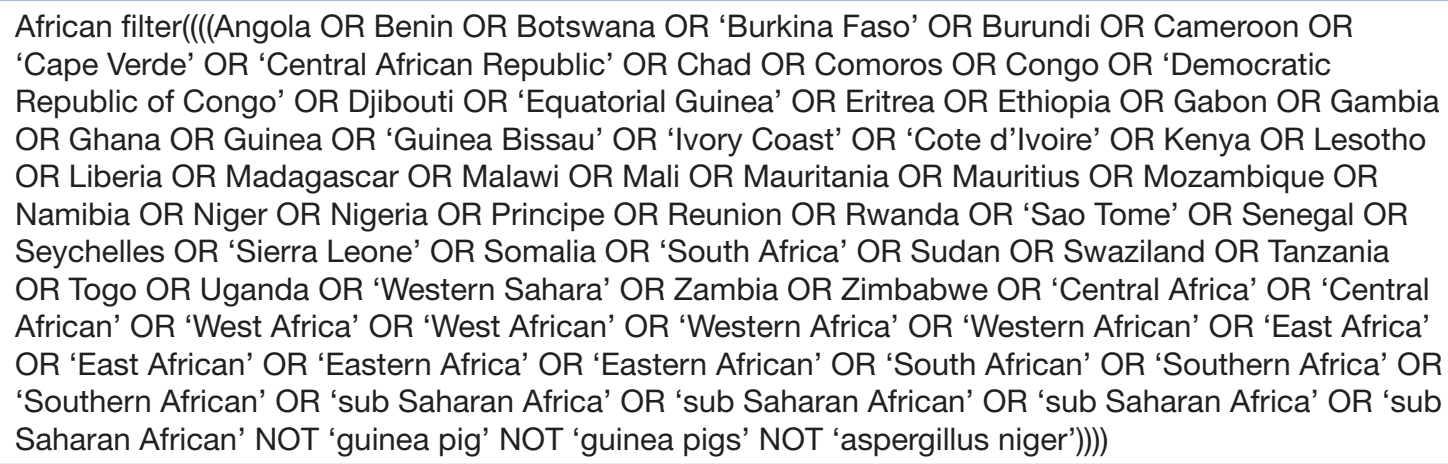 & \\
\hline$\# 7$ & \# 5 AND \# 6 Limits: 01/01/1990 to 28/02/2017 in English and French on humans & \\
\hline
\end{tabular}

HIV-infected and HIV-uninfected populations in SSA will be sought. The search retrieving will be broad, robust and precise using relevant medical subject headings $(\mathrm{MeSH})$ terms in combination with the African search filter, as indicated in table $3 .{ }^{59}$

1. Electronic search: The following databases will be searched: PubMed/Medline, EBSCOhost, Web of Science, Google Scholar, Scopus, African Index Medicus and Cochrane Database of Systematic Reviews for eligible studies.

2. Reference lists search: The reference lists of relevant material will be searched to identify additional studies of interest.

3. Grey literature search: Authors, experts in the field and authors of conference proceedings will be contacted through emails for any relevant information, data and results. Studies will be excluded after three unsuccessful attempts to contact the author.

4. Search management: The records of retrieved articles will be managed using EndNote Reference Manager $\mathrm{X} .{ }^{60}$ The included and excluded articles at each screening stage will be stored as different files. Figure $1^{61}$ indicates the prototype steps for managing the records and data for the review.

\section{Study screening and selection}

A screening criteria checklist will be developed using Google Forms, and tested for reliability and applicability to select relevant studies, and will involve three levels: title, abstract and full article screening.

1. Title screening: One investigator will independently select studies that meet the inclusion criteria by screening article titles for significance to the review focus and outcome, and duplicate citations will be excluded.

2. Abstract screening: Four investigators, two reviewers per language, will independently review abstracts of the included title-screened articles, being guided by the stated inclusion criteria.

3. Full article screening: This will be similar to the abstract screening and will further establish the eligibility of the identified studies. The first reviewer will assess for the articles' eligibility for meta-analysis, general characteristics and outcomes. The second reviewer will verify at least $50 \%$ of the studies for general characteristics information and $100 \%$ of studies for outcomes data.

4. Screening agreement and disagreement: Screening will establish the inter-rater reliability using Cohen's kappa coefficient, $\kappa$, which is a robust statistic used for inter-rater reliability testing, ${ }^{62} 63$ and any disagreement will be resolved through consultation of the study coordinator, if necessary. Authors will be contacted if there are missing information and data from the published articles that are relevant to the study and for further reported result clarity where needed. The reasons for exclusion at all stages will be documented.

\section{Data extraction}

A Google Form will be designed, pretested and standardised to extract data from the reviewed and included studies. The data extraction and entry will also be conducted by two reviewers to establish an inter-rater reliability and avoid data entering errors, ${ }^{63}$ with disagreement 


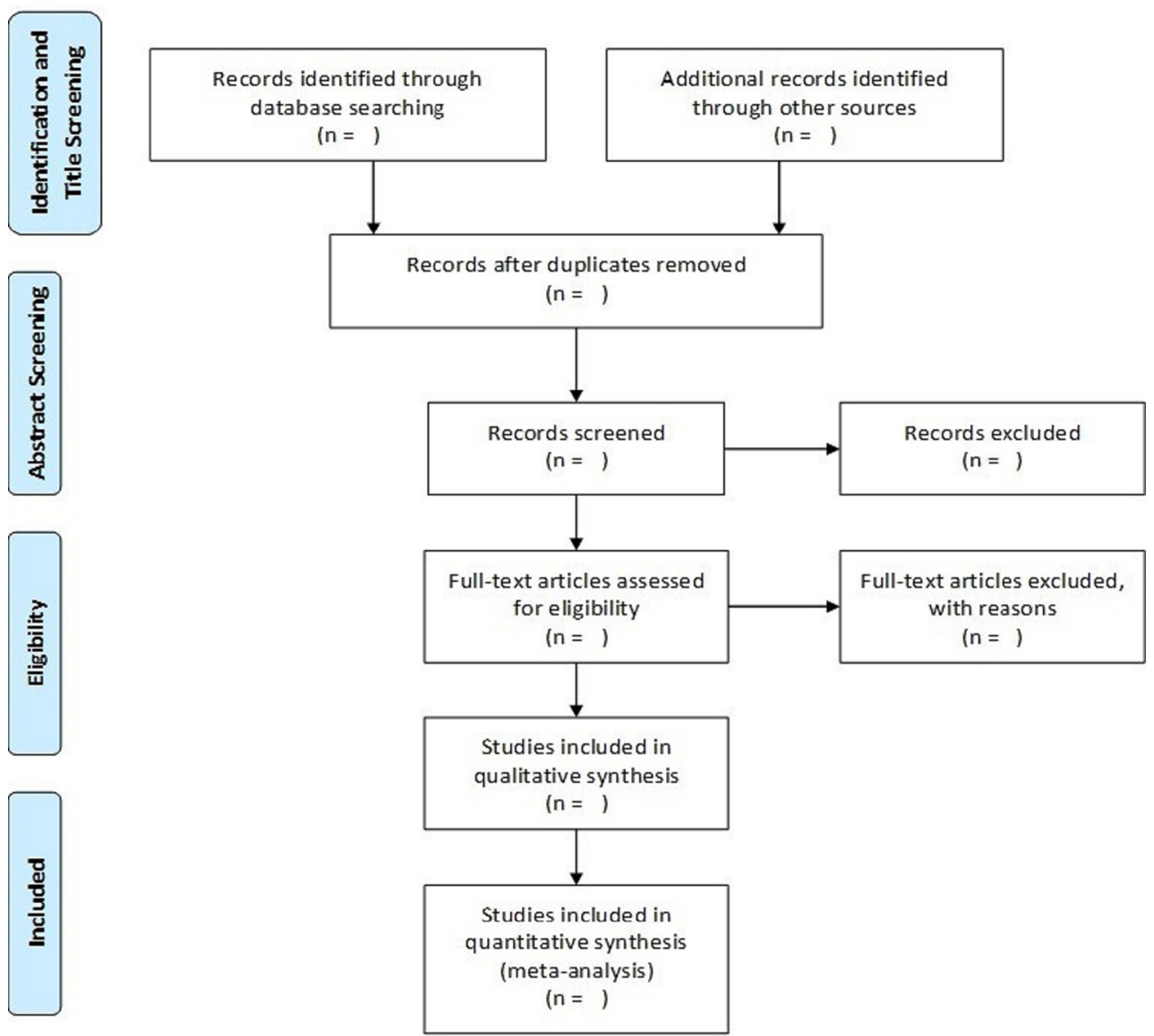

Figure 1 Search management flow chart for review.

resolved by the study coordinator. The following data items will be extracted:

1. Publication details: author(s) name, year of publication, year(s) of study, language, publication status.

2. Study characteristics and settings: rural or urban, study site, hospital or community/population-based country settings, study design, sample size, length of study duration of follow-up, source of funding, ethical approval.

3. Study participant's characteristics: sex proportion, mean age, HIV status, participants' number and proportion for single studies with multiple outcomes and/or population subgroups.

4. Study outcome: Mets, diabetes and hypertension, comorbid diabetes and hypertension.

5. Study target population: HIV-positive and/or HIVnegative study participants, number and proportion of participants with or comorbid study outcome (CI, $\mathrm{p}$ values).

\section{Quality and risk of bias assessment}

The quality assessment will be conducted by two reviewers using the Effective Public Health Practice Project/McMaster Evidence Review and Synthesis Centre Tool: Quality Assessment Tool for Quantitative Studies (see online supplementary appendix 1). ${ }^{64}$ The tool was selected due to its use of summary scoring to determine the quality of studies, ranging from strong, moderate to weak, and its ability to assess all types of quantitative study methods. This is important, as scale or checklist tools are more likely to include criteria that do not directly relate to internal validity, resulting in unreliable validity assessment. ${ }^{65}$ The summary scores for all the included studies will be documented and reported in the final review. The study's risk of bias will be performed using the risk of bias tool for prevalence studies by Hoy et $a l^{66}$ and the Cochrane guidelines available in Review Manager V.5.3 (http://tech.cochrane.org/revman) (table 4). The quality and risk of bias assessment will be presented as part of the table of characteristics of the included studies. The inter-rater agreement will be calculated using the proportion of agreement and kappa statistics. The minimum sample size for included studies will be calculated to determine a good precision estimate, using the pooled estimate of study outcomes prevalence among PLHIV and HIV-negative populations. This calculation will use the Clopper-Pearson CI formula, ${ }^{67}$ and a study with good precision for this meta-analysis will be defined as one whose sample size is greater than or equal to the calculated minimum sample size. 
Table 4 Risk of bias tool (adapted from Hoy et al ${ }^{66}$ tool for prevalence studies)

\section{Study title: \\ Name of author(s) \\ Year of publication}

Risk of bias items

Risk of bias level: low $\quad$ yes $=0$,

External validity

1. Was the study target population a close representation of the national population in

relation to relevant variables, for example, age, sex?

2. Was the sampling frame a true or close representation of the target population?

3. Was some form of random selection used to select the sample, OR, was a census undertaken?

4. Was the likelihood of non-participation bias minimal?

Internal validity

5. Was data collected directly from the participants (as opposed to proxy)?

6. Were acceptable case definitions and diagnostic measures of metabolic syndrome, diabetes and hypertension used?

7. Were the study instruments that measured the parameter of interest (eg, prevalence of diabetes) shown to have reliability and validity (if necessary)?

8. Was the same mode of data collection used for all study participants?

9. Was the length of the shortest prevalence period for the parameter of interest appropriate?

10. Were the numerator(s) and denominator(s) for the calculation of the prevalence of

metabolic syndrome, diabetes and hypertension appropriate?

Presentation of summary on the overall risk of study bias.

The total score ranged from 0 to 9 , with the overall score categorised as follows:

1. Low risk of bias: 8 or more 'yes' answers, further research is very unlikely to change our confidence in the estimate.

2. Moderate risk of bias: 6-7 'yes' answers, further research is likely to have an important impact on our confidence and may change in the estimate.

3. High risk of bias: 5 or fewer 'yes' answers, further research is very likely to have an important impact on our confidence and likely change the estimate.

\section{Data synthesis and analysis}

The data will be systematically described, analysed and summarised to answer the four research questions, and the data will be narratively synthesised if meta-analysis cannot be performed.

\section{Data analysis}

The prevalence results of Mets (and its subcomponents), discrete and/or comorbid diabetes and hypertension will be stratified and compared by HIV status to establish any significant difference. As some definitions of Mets include diabetes and blood pressure as criteria, the prevalence of subcriteria in studies that assessed Mets across HIV-positive and HIV-negative populations will be included as a secondary objective. The prevalence estimate will be presented by country, geographical region and HIV status. Furthermore, given the international changes in the definition of diabetes, hypertension and Mets, the analysis will also be stratified by the period of definition used in the included/eligible studies. This study-specific prevalence estimates will be pooled using the random-effects meta-analysis model to present the mean of the distribution of effects between HIV-infected and HIV-negative populations. ${ }^{68}$ The variance for the random meta-analysis estimate will be computed using the updated DerSimonian and Laird variance estimator method. ${ }^{69}$ The random-effects model will be used in anticipation of substantial variation in Mets prevalence and on the different outcome definitions across the included studies/period. To minimise the effect of extreme prevalence on the overall estimates, single arcsine transformation will be performed on the raw prevalence before pooling the data. ${ }^{70}$ CIs at $95 \%$ will be calculated for all reported study outcome prevalence measures using the Clopper-Pearson method. ${ }^{67}$

\section{Heterogeneity}

The statistical heterogeneity in the meta-analysis will be assessed using the $\mathrm{I}^{2}$ statistic, ${ }^{71}$ and if the $\mathrm{I}^{2}$ value is greater than $50 \%$, it will be regarded as substantial heterogeneity. Forest plots and the overall random-effects pooled estimate will be generated to display prevalence with the corresponding CI for each study. If asymmetry is present based on visual assessment, exploratory analyses will be performed to investigate and adjust this using trim and/or fill analysis. The prevalence of 
the study outcomes between mean/median age and gender will be estimated. Meta-regression analysis will be explored among HIV-infected participants who are on antiretroviral treatment and are treatment-naïve, if adequate data are found.

\section{Publication bias}

If there are $\geq 10$ studies in the meta-analysis, publication bias will be further investigated using funnel plots and Egger's test, ${ }^{72}$ the data being analysed using Stata V.13.0.

\section{Sensitivity analysis}

A sensitivity analysis to assess the robustness of the individual study designs and data set of the observed outcomes to the analysis assumptions will also be undertaken to further reduce the risk of bias assessment. The primary analysis will be repeated with altered extracted data sets and statistical methods to determine any changes in the combined outcome estimate effect. ${ }^{73}$ The data set and statistical model for the statistical analysis will be determined during the review process and reported in the final review.

\section{Strength of evidence assessment}

The quality and strength of evidence extracted from the included and analysed studies will be assessed using the Grading of Recommendations Assessment, Development and Evaluation (GRADE) approach. The GRADEpro software will be used to assist in grading the evidence and presenting the summary of findings in the review and meta-analysis. $^{74}$

\section{Review reporting and dissemination}

The proposed systematic review and meta-analysis will be guided by the PRISMA guidelines. ${ }^{58}$ The final report and completed PRISMA checklist will be published as a scientific article in a peer-reviewed journal. In addition, the review findings will be presented at conferences and/or to relevant health agencies.

\section{Potential amendments}

While there are no planned amendments for this protocol, if any substantial amendments arise during the review itself, these will be documented and reported in the published findings.

\section{Ethical consideration}

As the present review and meta-analysis study will use aggregated published data and information for analysis, no ethical approval will be required.

\section{CONCLUSION}

This systematic review and meta-analysis will attempt to identify the distribution of Mets, diabetes and hypertension and their common related comorbidities between PLHIV and HIV-negative general populations across SSA. The rigorous methodology proposed for this review will ensure a robust knowledge synthesis and provide evidence-based knowledge of the prevalence of the study outcomes for stakeholders, researchers and policy makers. This will assist with indicating the research gaps and priorities for Mets, diabetes and hypertension in the whole of Africa.

Acknowledgements Open access for this publication has been made possible through support from the Victor Daitz Information Gateway, an initiative of the Victor Daitz Foundation and the University of KwaZulu-Natal.

Contributors $00 \mathrm{~T}$ and BS were responsible for manuscript conceptualisation. 00T was responsible for initial manuscript drafting, with BS contributing to the revision and editing of the manuscript. OOT and BS read and approved the final manuscript. 00T is the guarantor of the review.

Funding College of Health Sciences Scholarship.

Competing interests None declared.

Patient consent The study is a systematic review and meta-analysis and does not involve patients.

Provenance and peer review Not commissioned; externally peer reviewed.

Open Access This is an Open Access article distributed in accordance with the Creative Commons Attribution Non Commercial (CC BY-NC 4.0) license, which permits others to distribute, remix, adapt, build upon this work non-commercially, and license their derivative works on different terms, provided the original work is properly cited and the use is non-commercial. See: http://creativecommons.org/ licenses/by-nc/4.0/

(c) Article author(s) (or their employer(s) unless otherwise stated in the text of the article) 2017. All rights reserved. No commercial use is permitted unless otherwise expressly granted.

\section{REFERENCES}

1. Omran AR. The epidemiologic transition: a theory of the epidemiology of population change. 1971. Milbank $Q$ 2005;83:731-57.

2. Agyei-Mensah $S$, de-Graft Aikins A. Epidemiological transition and the double burden of disease in Accra, Ghana. J Urban Health 2010;87:879-97.

3. Frenk J, Bobadilla JL, Sepuúlveda J, et al. Health transition in middle-income countries: new challenges for health care. Health Policy Plan 1989;4:29-39.

4. Demographic KDB. Epidemiological. and health transitions: are they relevant to population health patterns in Africa? 2014;2014:7.

5. Tabutin D, Schoumaker B, Rabenoro M. The demography of Sub-Saharan Africa from the 1950 s to the 2000s. Population 2004;59:455-622.

6. Lurie MN, Rosenthal S. Concurrent partnerships as a driver of the HIV Epidemic in sub-Saharan Africa? The evidence is limited. AIDS Behav 2010;14:17-24.

7. Organization WH. Burden: Mortality, morbidity and risk factors. Global status report on non communicable diseases , 2010.

8. Dalal S, Beunza JJ, Volmink J, et al. Non-communicable diseases in sub-Saharan Africa: what we know now. Int J Epidemiol 2011;40:885-901.

9. Zimmet P, Magliano D, Matsuzawa Y, et al. The metabolic syndrome: a global public health problem and a new definition. $J$ Atheroscler Thromb 2005;12:295-300.

10. Alberti K, Davidson MB, DeFronzo RA, et al. Report of the expert committee on the diagnosis and classification of diabetes mellitus. Diabetes care 1998;21:S5

11. Rahimi K, Emdin CA, MacMahon S. The epidemiology of blood pressure and its worldwide management. Circ Res 2015;116:925-36.

12. Zimmet PZ, Alberti KG. Epidemiology of Diabetes-Status of a Pandemic and Issues Around Metabolic Surgery. Diabetes Care 2016;39:878-.

13. Kearney PM, Whelton M, Reynolds K, et al. Global burden of hypertension: analysis of worldwide data. Lancet 2005;365:217-23.

14. Shaw JE, Sicree RA, Zimmet PZ. Global estimates of the prevalence of diabetes for 2010 and 2030. Diabetes Res Clin Pract 2010;87:4-14.

15. Zimmet $P$, Alberti KG, Magliano DJ, et al. Diabetes mellitus statistics on prevalence and mortality: facts and fallacies. Nat Rev Endocrinol 2016;12:616-22.

16. Wild S, Roglic G, Green A, et al. Global prevalence of diabetes: estimates for the year 2000 and projections for 2030. Diabetes Care 2004;27:1047-53. 
17. Adler Al, Stratton IM, Neil HA, et al. Association of systolic blood pressure with macrovascular and microvascular complications of type 2 diabetes (UKPDS 36): prospective observational study. BMJ 2000;321:412-9.

18. Mozaffarian D. Dietary and Policy Priorities for Cardiovascular Disease, Diabetes, and Obesity. A Comprehensive Review 2016;133:187-225.

19. Assah FK, Ekelund U, Brage S, et al. Urbanization, physical activity, and metabolic health in sub-Saharan Africa. Diabetes Care 2011;34:491-6.

20. Kassi E, Pervanidou P, Kaltsas G, et al. Metabolic syndrome: definitions and controversies. BMC Med 2011:9:1.

21. Okafor $\mathrm{Cl}$. The metabolic syndrome in Africa: Current trends. Indian $J$ Endocrinol Metab 2012;16:56

22. Motala AA, Mbanya JC, Ramaiya KL. Metabolic syndrome in subSaharan Africa. Ethn Dis 2009;19(Suppl 2):S2-8.

23. Duvnjak L, Bulum T, Metelko Z. Hypertension and the metabolic syndrome. Diabetologia Croatica 2008;37:83-9.

24. Parikh RM, Mohan V. Changing definitions of metabolic syndrome. Indian J Endocrinol Metab 2012;16:7-12.

25. Eckel RH, Kahn SE, Ferrannini E, et al. Obesity and type 2 diabetes: what can be unified and what needs to be individualized? Diabetes Care 2011;34:1424-

26. Bosello O, Zamboni M. Visceral obesity and metabolic syndrome. Obes Rev 2000;1:47-56.

27. Furukawa S, Fujita T, Shimabukuro M, et al. Increased oxidative stress in obesity and its impact on metabolic syndrome. J Clin Invest 2004;114:1752-61

28. Ipadeola A, Adeleye JO. THE metabolic syndrome and accurate cardiovascular risk prediction in persons with type 2 diabetes mellitus. Diabetes Metab Syndr 2016;10:7-12.

29. Gazzaruso C, Bruno R, Garzaniti A, et al. Hypertension among HIV patients: prevalence and relationships to insulin resistance and metabolic syndrome. J Hypertens 2003;21:1377-82.

30. Wand H, Calmy A, Carey DL, et al. Metabolic syndrome, cardiovascular disease and type 2 diabetes mellitus after initiation of antiretroviral therapy in HIV infection. AIDS 2007;21:2445-53.

31. Paula AA, Falcão MC, Pacheco AG. Metabolic syndrome in HIVinfected individuals: underlying mechanisms and epidemiological aspects. AIDS Res Ther 2013;10:32.

32. Sweet DE. Metabolic complications of antiretroviral therapy. Top HIV Med 2005;13:70-4.

33. Park YW, Zhu S, Palaniappan L, et al. The metabolic syndrome: prevalence and associated risk factor findings in the US population from the Third National Health and Nutrition Examination Survey, 1988-1994. Arch Intern Med 2003;163:427-36.

34. Nguyen KA, Peer N, Mills EJ, et al. Burden, determinants, and pharmacological management of hypertension in HIV-Positive patients and populations: a systematic narrative review. AIDS Rev 2015;17:83-95

35. Nguyen KA, Peer N, Mills EJ, et al. A Meta-Analysis of the Metabolic Syndrome Prevalence in the Global HIV-Infected Population. PLoS One 2016;11:e0150970.

36. Yang HI, Kim HC, Jeon JY. The association of resting heart rate with diabetes, hypertension, and metabolic syndrome in the Korean adult population: The fifth Korea National Health and Nutrition Examination Survey. Clin Chim Acta 2016;455:195-200.

37. Laaksonen DE, Lakka HM, Niskanen LK, et al. Metabolic syndrome and development of diabetes mellitus: application and validation of recently suggested definitions of the metabolic syndrome in a prospective cohort study. Am J Epidemiol 2002;156:1070-7.

38. Glass RI. HIV/AIDS and noncommunicable disease comorbidities: emerging research priorities. J Acquir Immune Defic Syndr 2014;67(suppl 1):S1.

39. Adeloye D, Basquill C. Estimating the prevalence and awareness rates of hypertension in Africa: a systematic analysis. PLOS One 2014:9:e104300.

40. Cappuccio FP, Miller MA. Cardiovascular disease and hypertension in sub-Saharan Africa: burden, risk and interventions. Intern Emerg Med 2016;11:299-305.

41. Angkurawaranon C, Nitsch D, Larke N, et al. Ecological Study of HIV Infection and Hypertension in Sub-Saharan Africa: Is There a Double Burden of Disease? PLoS One 2016;11:e0166375.

42. Dillon DG, Gurdasani D, Riha J, et al. Association of HIV and ART with cardiometabolic traits in sub-Saharan Africa: a systematic review and meta-analysis. Int J Epidemiol 2013;42:1754-71.

43. Naidu S, Ponnampalvanar S, Kamaruzzaman SB, et al. Prevalence of metabolic syndrome among people living with HIV in developing countries: a systematic review. AIDS Patient Care STDS 2017;31:1-13.
44. Islam FM, Wu J, Jansson J, et al. Relative risk of cardiovascular disease among people living with HIV: a systematic review and metaanalysis. HIV Med 2012;13:n/a-68.

45. Mottillo S, Filion KB, Genest J, et al. The metabolic syndrome and cardiovascular risk a systematic review and meta-analysis. J Am Coll Cardiol 2010;56:1113-32.

46. Young F, Critchley JA, Johnstone LK, et al. A review of co-morbidity between infectious and chronic disease in Sub Saharan Africa: TB and diabetes mellitus, HIV and metabolic syndrome, and the impact of globalization. Global Health 2009;5:9.

47. Nduka CU, Stranges S, Sarki AM, et al. Evidence of increased blood pressure and hypertension risk among people living with HIV on antiretroviral therapy: a systematic review with meta-analysis. J Hum Hypertens 2016;30:355-62.

48. Lloyd-Sherlock P. Population ageing in developed and developing regions: implications for health policy. Soc Sci Med 2000;51:887-95.

49. Hilderink HB, Plasmans MH, Snijders BE, et al. Accounting for multimorbidity can affect the estimation of the Burden of Disease: a comparison of approaches. Arch Public Health 2016;74:37

50. Maher D, Ford N, Unwin N. Priorities for developing countries in the global response to non-communicable diseases. Global Health 2012;8:14.

51. Ntuli ST, Maimela E, Alberts M, et al. Prevalence and associated risk factors of hypertension amongst adults in a rural community of Limpopo Province, South Africa. Afr J Prim Health Care Fam Med 2015;7:847.

52. Organization WH. Definition and diagnosis of diabetes mellitus and intermediate hyperglycaemia:report of a WH, 2006.

53. Chobanian AV, Bakris GL, Black HR, et al. Seventh report of the Joint National Committee on Prevention, Detection, Evaluation, and Treatment of High Blood Pressure. Hypertension 2003;42:1206-52.

54. Alberti KG, Zimmet P, Shaw J. Metabolic syndrome--a new worldwide definition. A Consensus Statement from the International Diabetes Federation. Diabet Med 2006;23:469-80.

55. Grundy SM, Brewer HB, Cleeman JI, et al. Definition of metabolic syndrome.. Report of the National Heart, Lung, and Blood Institute/ American Heart Association Conference on Scientific Issues Related to Definition 2004;109:433-8.

56. Balkau B, Charles M-A. Comment on the provisional report from the WHO consultation. Diabetic medicine 1999;16:442-3.

57. Jüni P, Holenstein F, Sterne J, et al. Direction and impact of language bias in meta-analyses of controlled trials: empirical study. Int $J$ Epidemiol 2002;31:115-23.

58. Shamseer L, Moher D, Clarke M, et al. Preferred reporting items for systematic review and meta-analysis protocols (PRISMA-P) 2015: elaboration and explanation. BMJ 2015;349:g7647.

59. Pienaar E, Grobler L, Busgeeth K, et al. Developing a geographic search filter to identify randomised controlled trials in Africa: finding the optimal balance between sensitivity and precision. Health Info Libr J 2011;28:210-5.

60. Reiss M, Reiss G. EndNote 5 reference manager--functions-improvements--personal experiences. Praxis 2002;91:1645-50.

61. Moher D, Liberati A, Tetzlaff J, et al. Preferred reporting items for systematic reviews and meta-analyses: the PRISMA statement. PLOS Med 2009;6:e1000097.

62. McHugh ML. Interrater reliability: the kappa statistic. Biochem Med 2012;22:276-82.

63. Uman LS. Systematic reviews and meta-analyses. J Can Acad Child Adolesc Psychiatry 2011;20:57.

64. Methods NCCf T. Quality Assessment Tool for quantitative studies. McMaster University Hamilton: ON, 2008.

65. Higgins JP, Green S. Cochrane handbook for systematic reviews of interventions: John Wiley \& Sons, 2011.

66. Hoy $D$, Brooks $P$, Woolf $A$, et al. Assessing risk of bias in prevalence studies: modification of an existing tool and evidence of interrater agreement. J Clin Epidemiol 2012;65:934-9.

67. Vollset SE. Confidence intervals for a binomial proportion. Stat Med 1993;12:809-24.

68. Borenstein M, Hedges LV, Higgins J, et al; Front matter: Wiley Online Library, 2009.

69. DerSimonian R, Kacker R. Random-effects model for meta-analysis of clinical trials: an update. Contemp Clin Trials 2007;28:105-14.

70. Barendregt JJ, Doi SA, Lee YY, et al. Meta-analysis of prevalence. $J$ Epidemiol Community Health 2013;67:974-8.

71. Higgins JP, Thompson SG, Deeks JJ, et al. Measuring inconsistency in meta-analyses. BMJ 2003;327:557-60.

72. Borenstein M. Software for publication bias. Publication bias in metaanalysis: Prevention, assessment and adjustments 2005:193-220.

73. Bown MJ, Sutton AJ. Quality control in systematic reviews and metaanalyses. Eur J Vasc Endovasc Surg 2010;40:669-77. 
74. GRADEpro G. GRADEpro Guideline Development Tool (Software). McMaster University(developed by Evidence Prime, Inc): gradepro org. 2015.
75. Grundy SM, Cleeman JI, Daniels SR, et al. Diagnosis and management of the metabolic syndrome. Circulation 2005;112:2735-52. 